\title{
Temperature dependence of lower critical field of YBCO Superconductor
}

\author{
Poonam Rani ${ }^{1,2, a)}$, A.K. Hafiz ${ }^{2}$ and V.P.S. Awana ${ }^{1}$ \\ ${ }^{1}$ National Physical Laboratory (CSIR) Dr. K. S. Krishnan Road, New Delhi-110012, India \\ ${ }^{2}$ Department of Physics, Jamia Millia Islamia, New Delhi-110025, India \\ a) Corresponding author: jangrapoonam62@yahoo.com
}

\begin{abstract}
We report the detailed study of the temperature dependence of the lower critical field $\left(\mathrm{H}_{\mathrm{cl}}\right)$ of the $\mathrm{YBa}_{2} \mathrm{Cu}_{3} \mathrm{O}_{7}$ superconductor by magnetization measurements. The curve shows the multiband gap behavior of the sample. It is found that the sample is not a single BCS type superconductor. $\mathrm{H}_{\mathrm{cl}}$ is measured as the point at which the curve deviates from a Meissner-like linear $\mathrm{M}(\mathrm{H})$ curve to a nonlinear path. The $\mathrm{H}_{\mathrm{c} 1}$ for $\mathrm{YBCO}$ at different temperatures from $10 \mathrm{~K}$ to $85 \mathrm{~K}$ has been determined by magnetization measurements $\mathrm{M}(\mathrm{H})$ with applied field parallel to the c-axis. The sample phase purity has been confirmed by Rietveld fitted X-ray diffraction data. The amplitude (1-17Oe) dependent AC susceptibility confirms the granular nature of superconducting compound. Using Bean model we calculated the temperature dependency of intergrain critical current density and $\mathrm{J}_{\mathrm{c}}(0)$ is found as $699.14 \mathrm{kAcm}^{-2}$.
\end{abstract}

\section{INTRODUCTION}

Lower critical field $\left(\mathrm{H}_{\mathrm{cl}}\right)$, is an intrinsic parameter related to the mechanism of superconductivity in type -II superconductors. Measurements of $\mathrm{H}_{\mathrm{c} 1}$, the upper critical field, $\left(\mathrm{H}_{\mathrm{c} 2}\right)$ and coherence length $(\xi)$ give a complete description of the mixed state parameters of the type-II superconductor. The $\mathrm{H}_{\mathrm{cl}}$ is defined as the field at which magnetization curve starts deviating from its linear path or one can say that the field at which magnetic vortices first penetrate a type-II superconductor. Also, $\mathrm{H}_{\mathrm{cl}}$ is directly related to the free energy of a flux line and contains information regarding essential mixed state parameters, such as the penetration depth, $\lambda$ and the Ginzburg-Landau parameter, $\kappa$. Also, the multi-band superconducting gap can be measured from the $\mathrm{H}_{\mathrm{c} 1}$ and magnetic penetration depth $\lambda$. The $\mathrm{H}_{\mathrm{cl}}$ has already been determined by different methods as mentioned in earlier reported literature [1-4]. Hafiez et.al. determined $\mathrm{H}_{\mathrm{cl}}$ by two different methods i.e. the transition of field from a Meissner-like linear $\mathrm{M}(\mathrm{H})$ curve to a nonlinear path and second is by measuring the onset of the trapped moment Mt and a linear temperature dependence of lower critical filed was found. Some has characterized dc magnetization measurement by a Vibrating Sample Magnetometer (VSM) and Hall sensor methods [5-7]. D. X. Chen et. al. calculated $\mathrm{H}_{\mathrm{cl}}$ for $\mathrm{YBCO}$ compound from hysteresis loop by fitting the data with an extended critical state model. Moreover, The temperature dependence of $\mathrm{H}_{\mathrm{c} 1}$ data has been investigated and several results concluded that $\mathrm{H}_{\mathrm{cl}}(\mathrm{T})$ function does not follow BCS theory. Generally, it is expected that the temperature dependence lower critical field $\mathrm{H}_{\mathrm{cl}}$ follow Bardeen-Cooper-Schrieffer (BCS) theory. However, $\mathrm{H}_{\mathrm{cl}}(\mathrm{T})$ measurement consistently shows an upturn at $\mathrm{T}<\mathrm{T}_{\mathrm{c} / 2}$ when it does not obey the BCS theory, also popularly known as the non BCS behavior $[2,8,9]$.

In this paper we report the detailed magnetization measurements on YBCO superconductor. We studied the critical current density of YBCO sample from detailed AC magnetic susceptibility measurements. Also, the report includes the complete $\mathrm{M}(\mathrm{H})$ curve at $10 \mathrm{~K}$ and variation of $\mathrm{DC}$ magnetization with field at different temperatures $(10 \mathrm{~K}-85 \mathrm{~K})$. The data of $\mathrm{M}(\mathrm{H})$ curve permits a more precise determination of the lower critical field. The lower critical field was determined from the magnetization measurements using procedure based on first deviation from perfect diamagnetism. Further it explains the temperature dependence of the lower critical fields $\mathrm{H}_{\mathrm{cl}}$ and found a non BCS type behavior.

\footnotetext{
2nd International Conference on Condensed Matter and Applied Physics (ICC 2017)

AIP Conf. Proc. 1953, 120026-1-120026-4; https://doi.org/10.1063/1.5033091

Published by AIP Publishing. 978-0-7354-1648-2/\$30.00
} 


\section{EXPERIMENTAL}

The sample $\mathrm{YBa}_{2} \mathrm{Cu}_{3} \mathrm{O}_{7}$ is prepared through conventional solid state reaction route. After initial grinding, calcinations are done at $890^{\circ} \mathrm{C}$ and $905^{\circ} \mathrm{C}$ temperatures with intermediate grinding. Finally the sample is annealed with flowing oxygen at $920^{\circ} \mathrm{C}$ for $12 \mathrm{~h}, 600^{\circ} \mathrm{C}$ for $12 \mathrm{~h}$ and $450^{\circ} \mathrm{C}$ for $12 \mathrm{~h}$ and subsequently slow cooling is done to room temperature. The sample is characterized by the $\mathrm{X}$-ray powder diffraction technique using Rigaku X-ray diffractometer $(\mathrm{Cu}-\mathrm{K} \alpha$ line). Rietveld analysis of the sample is performed using Fullprof program. Detailed AC susceptibility, magnetization measurements and transport measurements are done on Physical Properties Measurement System (Quantum Design-USA PPMS-14T).

\section{RESULTS AND DISCUSSION}

Figure 1 shows the Rietveld fitted XRD pattern of synthesized YBCO sample. As no extra peaks of impurity are found so the sample is considered as homogeneous and pure. The synthesized sample is crystallized in

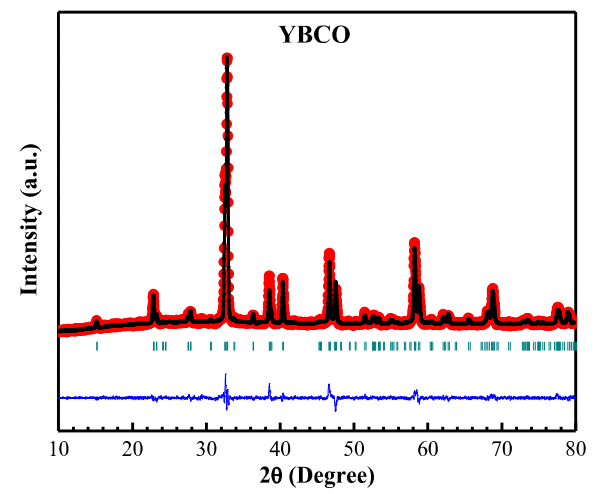

FIGURE 1: Rietveld fitted XRD pattern of YBCO, oxygen annealed sample.

orthorhombic structure within Pmmm space group. The fitted values of lattice parameters $a, b$ and $c$ are 3.823(2), $3.887(6)$ and 11.685(3) $\AA$, respectively. These values are close to oxygen stoichiometric $(\delta=0)$ YBCO compound. Rietveld fitting confirms the good quality of the synthesized sample.

Figure 2(a) exhibits the real part of AC susceptibility $(\chi /)$ at different (1Oe-170e) ac magnetic field amplitudes. It is important to note that the DC bias field is zero. The diamagnetic transition is observed at around 91.3K. Two step transition is very clear even on applying a small field amplitude of $1 \mathrm{Oe}$, corresponds to flux removal from intra

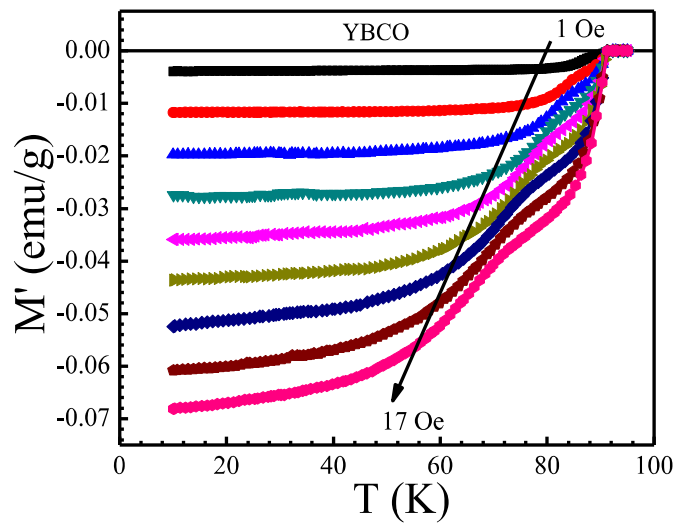

(a)

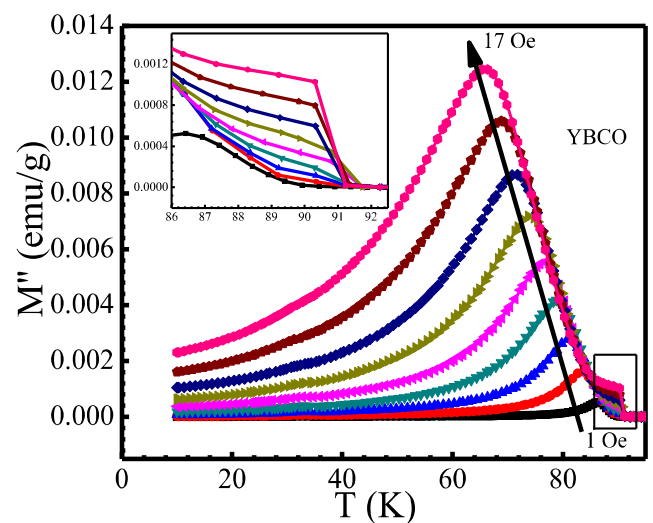

(b)

FIGURE 2: (a) Real part and (b) imaginary part of AC linear susceptibility measurements of YBCO taken at different ac field amplitudes with $333 \mathrm{~Hz}$ constant frequency. 
and inter grain region of the YBCO superconductor. This defines the granular nature of the studied sample. The first transition near $\mathrm{T}_{\mathrm{c}}$ i.e. intra granular transitions is observed at $91.3 \mathrm{~K}$ and transition at lower temperature is inter granular transition which is found at around $87.4 \mathrm{~K}$. The intra grain transition remains unaffected under high magnetic field amplitude but on applying high field the inter granular transition moves toward lower temperature. On varying magnetic field from $1 \mathrm{Oe}$ to $17 \mathrm{Oe}$, the inter granular transition shift from $87.4 \mathrm{~K}$ to $78.4 \mathrm{~K}$.

Figure 2(b) shows the imaginary part of the AC susceptibility $(\chi / /)$ for YBCO sample and the inset part is the clear view of intra granular peak. Similar to real part, clearly two peaks are observed in the $\chi / /$ versus $\mathrm{T}$ plot being taken at various AC amplitudes (1Oe-17Oe). The first peak (intra) occurs at around 90K and second peak (inter) at around $86.5 \mathrm{~K}$ with AC amplitude of 1Oe. With an increase in AC amplitude from 1Oe to 17Oe, the first peak though remains unaltered at around $90 \mathrm{~K}$, the second peak has a shift of $20.6 \mathrm{~K}$ towards lower temperature. This clearly indicates that though intra grain $T_{c}$ is unaltered with $A C$ amplitude, the inter grain $T_{c}$ is decreased significantly with field. Using Bean model [11] we calculated the temperature dependency of inter-grain critical current density. The relation is given by

$$
J_{c}\left(T_{P}\right)=H_{a} / \sqrt{ } a b
$$

Here, $2 \mathrm{a} \times 2 \mathrm{~b}$ where $\mathrm{a}<\mathrm{b}$, is cross section of sample, $\mathrm{H}_{\mathrm{a}}$, is applied ac field amplitude and $\mathrm{J}_{\mathrm{c}}\left(\mathrm{T}_{\mathrm{P}}\right)$ is the intergranular critical current density at $T_{P}$ and $T_{P}$ is the temperature of the inter-grain peak. The calculated values are plotted with temperature in Fig. 3(a). $\mathrm{J}_{\mathrm{c}}$ is estimated in the neighborhood of $\mathrm{T}_{\mathrm{c}}$, where the shift of the inter-grain peak's extendibility towards lower temperatures is within the limit of driven field. The temperature dependence of $\mathrm{J}_{\mathrm{c}}$ is found to obey power law given by [12]

$$
J_{c}(T)=J_{c}(0)\left(1-T_{p} / T_{c}\right)^{n}
$$

Here, $T_{c}$ is the zero resistivity critical temperature. Values of $J_{c}(0)$ and ' $n$ ' at $333 \mathrm{~Hz}$ obtained from experimental fitted data are $699.24 \mathrm{kAcm}^{-2}$ and 0.9 respectively which is in agreement with ref.[13]. The obtained value of $\mathrm{J}_{\mathrm{c}}(0)$ is quite high. The inter-granular current density obtained by using equation (2) is plotted in Fig. 3(b).

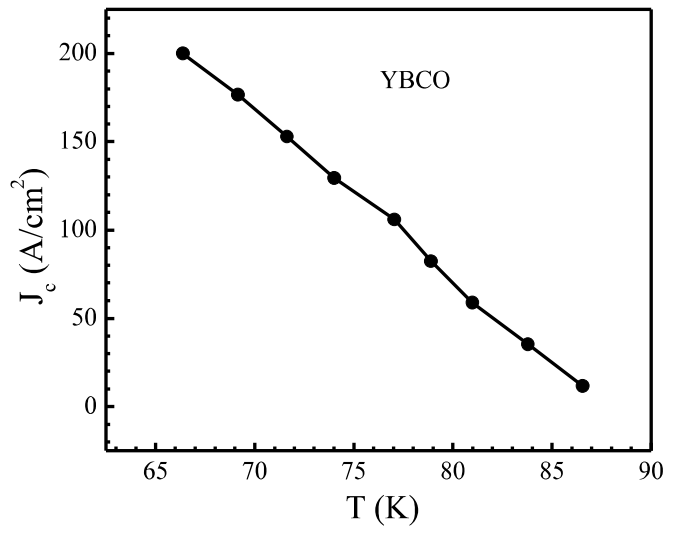

(a)

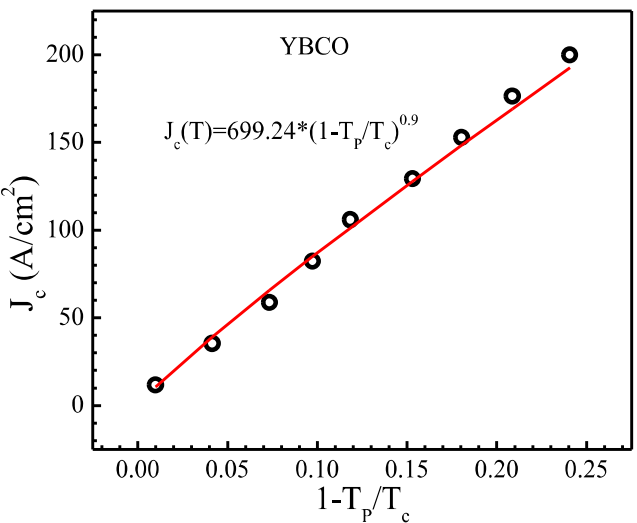

(b)

FIGURE 3: (a) Variation of $J_{c}$ with respect to $T_{P}$ of YBCO sample. (b) Temperature dependence of critical current density (symbols) and its fitted curve (solid line) as per equation (2) for YBCO sample.

In Figure 4, we present the magnetic hysteresis loop of YBCO sample at $10 \mathrm{~K}$ with the varying applied field. It is well known that the width and behavior of this hysteresis loop is the clear indication of the bulk critical current density in type II superconductors. The wider the hysteresis loop, the higher the bulk critical current density will be. This is in agreement with the calculated value of $J_{c}$ of the studied sample. Inset (a) of Fig. 4 shows the variation of DC magnetization with field $(\mathrm{M}-\mathrm{H})$ at several temperatures $(10 \mathrm{~K}$ to $85 \mathrm{~K})$ below superconducting transition temperature $\left(\mathrm{T}_{\mathrm{c}}\right)$. Now we calculate the lower critical field $\left(\mathrm{H}_{\mathrm{cl}}\right)$ from $\mathrm{M}-\mathrm{H}$ curves by using most popular method in which the estimated value of $\mathrm{H}_{\mathrm{c} 1}$ is the point from where the linear curve deviate its path towards non linear curve. It is seen that with increasing temperature, the $\mathrm{M}-\mathrm{H}$ curve shifts towards the zero value i.e. the value of lower critical field decreases with increase in temperature towards $\mathrm{T}_{\mathrm{c}}$. The variation of lower critical field $\left(\mathrm{H}_{\mathrm{c} 1}\right)$ with temperature is shown in Inset (b). This curve fitted in a general equation of lower critical field i.e. $\mathrm{H}_{\mathrm{cl}}(\mathrm{T})=\mathrm{H}_{\mathrm{cl}}(0) *\left(1-\left(\mathrm{T} / \mathrm{T}_{\mathrm{c}}\right)^{2}\right)$. Unlike to some other 
reports $[9,10]$ our data, $\mathrm{H}_{\mathrm{cl}}(\mathrm{T})$ doesn't obey the general equation of BCS theory. At low temperature value the curve completely diverts from BCS type behavior.

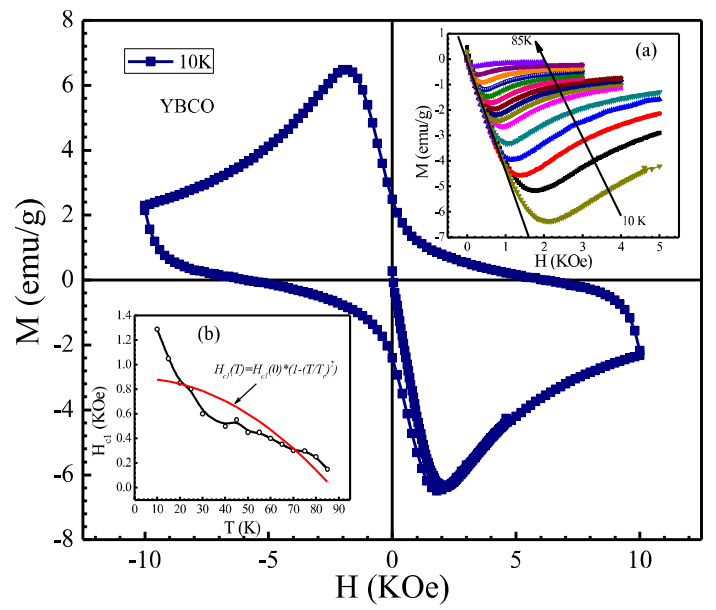

FIGURE 4: $\mathrm{M}-\mathrm{H}$ plot of $\mathrm{YBa}_{2} \mathrm{Cu}_{3} \mathrm{O}_{7}$ sample with temperature at $10 \mathrm{~K}$. Inset (a) Magnetic field dependent magnetization of $\mathrm{YBa}_{2} \mathrm{Cu}_{3} \mathrm{O}_{7}$ sample with temperature (b) Variation of lower critical field $\mathrm{H}_{\mathrm{cl}}$ with temperature and solid line represents the BCS fit.

It happens due to the existence of Fermi Surfaces (FSs) of different sizes and anisotropies [14]. This result is in good agreement with other reports [2,8]. This behavior may be the sign of multiband nature of superconductivity [5,7,14].

\section{CONCLUSION}

In summary, we extracted critical current density through magnetization measurement and it is found as $699.24 \mathrm{kAcm}^{-2}$. Also, we measured the complete hysteresis loop of a polycrystalline YBCO sample at $10 \mathrm{~K}$ and studied the $\mathrm{H}_{\mathrm{c} 1}$ as functions of temperature. The obtained data were fitted with high temperature BCS equation and found that $\mathrm{H}_{\mathrm{cl}}(\mathrm{T})$ function does not follow BCS theory. The sample shows multiband nature of superconductivity.

\section{ACKNOWLEDGEMENTS}

Poonam Rani would like to acknowledge CSIR for providing Senior Research Fellowship as a financial assistance. Authors like to thank Dr. D. K. Aswal, Director NPL for her keen interest in this work.

\section{REFERENCES}

1. C. Xiaowen, H. Sunli, L. Hongbao, Z. Weijie, Z. Yuheng, Cryogenics 29, 961-962, (1989).

2. D. -X. Chen, R. B. Goldfarb, R. W. Cross, A. Sanchez, Phys. Rev. B 48, 6426-6430, (1993).

3. V. Buntar, M. Ricco, L. Cristofolini, H. W. Weber, F. Bolzoni, Phys. Rev. B 52, 4432-4437, (1995).

4. T. Koyama, N. Takezawa, M. Tachiki, Physica C 168, 69-78, (1990).

5. M. A. Hafiez, J. Ge, A. N. Vasiliev, D. A. Chareev, J. V. Vondel, V. V. Moshchalkov, A. V. Silhanek, Phys. Rev. B 88, 174512-174520, (2013).

6. C. Ren, Z. -S. Wang, H. -Q. Luo, H. Yang, L. Shan, H. -H. Wen, Physica C 469, 599-605, (2009).

7. T. Shibauchi, K. Hashimoto, R. Okazaki, Y. Matsuda, Physica C 469, 590-601, (2009).

8. W. N. Hardy, D. A. Bonn, D. C. Morgan, R. Liang, K. Zhang, Phys. Rev. Lett. 70, 3999-4002, (1993).

9. D. H. Wu, S. Sridhar, Phys. Rev. Lett. 65, 2074-2077, (1990).

10. S. Sridhar, D. H. Wu, W. Kennedy, Phys. Rev. Lett. 63, 1873-1876, (1989).

11. C. P. Bean, Rev. Mod. Phys. 36, 31-39 (1964).

12. P. W. Anderson, Y. B. Kim, Rev. Mod. Phys. 36, 39-43 (1964).

13. A.J. B. -Leyva, R. Cobas, E. E. -Rams, M.T.D. Orlando, C. Noda, E. Altshuler, Physica C 331, 57-66, (2000).

14. Y. J. Song, J. S. Ghim, J. H. Yoon, K. J. Lee, M. H. Jung, H. S. Ji, J. H. Shim, Y. Bang, Y. S. Kwon, EPL 94, 57008-p6 (2011). 